\title{
CMEARTICLE
}

\section{Clinics in diagnostic imaging (203)}

Julian Sau Lian Chieng ${ }^{1}$, MD, FRCR, Kheng Song Leow ${ }^{1}$, MD, FRCR, Tze Chwan Lim ${ }^{1}$, MBBS, FRCR
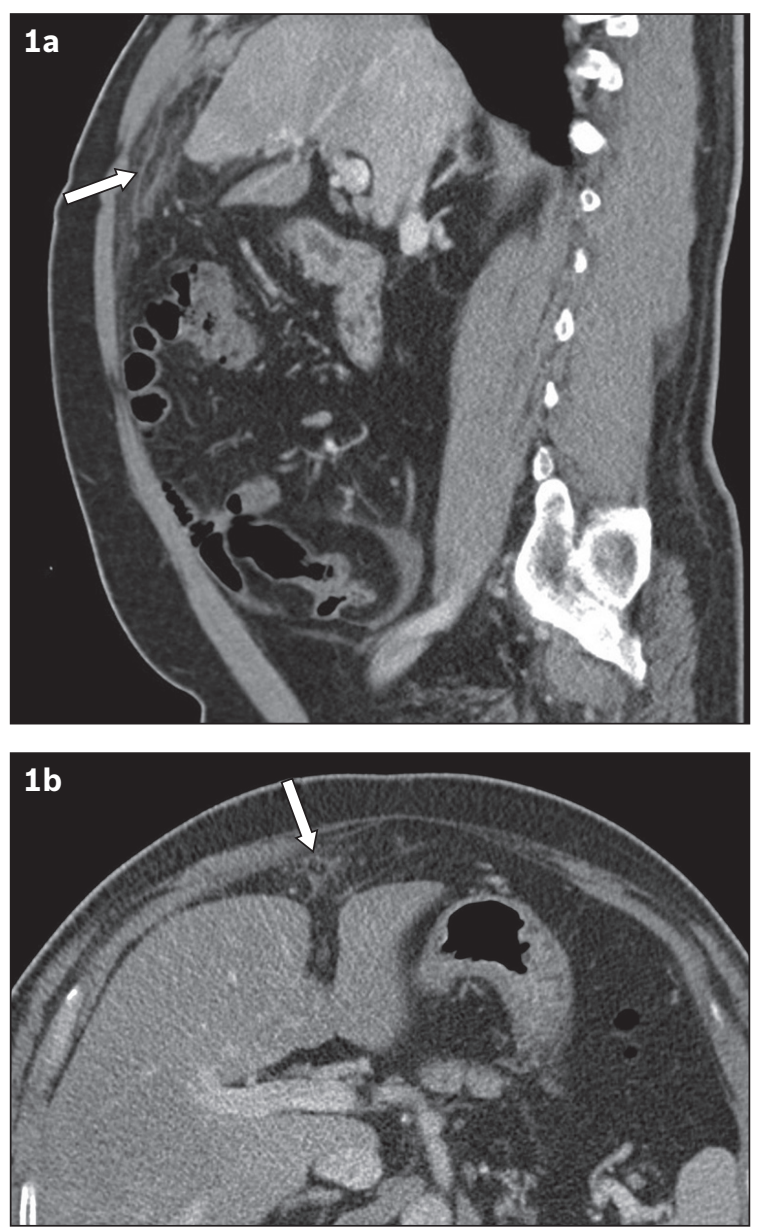

Fig. 1 (a) Sagittal and (b) axial contrast-enhanced CT image of the abdomen and pelvis.

\section{CASE PRESENTATION}

A 56-year-old man presented to the emergency department (ED) with a three-day history of pain in the epigastric and right hypochondrial regions of the abdomen. The pain was aggravated by deep inspiration and movement. There was no nausea, vomiting or associated gastrointestinal symptom. Physical examination revealed tenderness in the same regions as reported. There was no guarding or rebound tenderness. The patient had normal vital signs. Laboratory tests revealed mild leucocytosis of $11.33 \times 10^{9} / \mathrm{L}$ (normal range 3.82-9.91 $\times 10^{9} / \mathrm{L}$ ). Liver function test and serum amylase and renal panel showed normal results. An erect chest radiograph showed no subphrenic free air. Intravenous tramadol was administered for pain relief, but the pain persisted despite a period of observation. Computed tomography (CT) of the abdomen and pelvis (Fig. 1) was subsequently performed to evaluate for acute abdomen. What do these images show? What is the diagnosis? 


\section{IMAGE INTERPRETATION}

The sagittal contrast-enhanced CT image of the abdomen and pelvis (Fig. 1a) shows an ovoid area of fat attenuation with highattenuated peripheral rim (the 'hyperattenuating rim' sign) and surrounding inflammatory changes in the anterior perihepatic space, in close proximity to the falciform ligament (arrow). The axial contrast-enhanced CT image of the abdomen and pelvis (Fig. 1b) in the same patient shows the additional finding of a central, high-attenuated dot (arrow) within the area of encapsulated fat necrosis (the 'central dot' sign).

\section{DIAGNOSIS}

Focal infarction of the falciform ligament fatty appendage.

\section{CLINICAL COURSE}

The patient was managed conservatively. He was prescribed oral etoricoxib, a nonsteroidal anti-inflammatory drug (NSAID). The pain improved after NSAID administration and his vital signs remained normal. He was subsequently discharged with a prescription of a different oral NSAID, diclofenac, and was followed up in the outpatient surgery clinic.

\section{DISCUSSION}

Intraperitoneal focal fat infarction (IFFI) has been used to describe focal lipomatous tissue necrosis in various anatomical locations. ${ }^{(1-3)}$ The more common causes of IFFI include epiploic appendagitis and omental infarction. These conditions have been extensively reported in the literature. However, focal infarction of the fatty appendage of the falciform ligament is extremely rare, with less than 20 cases reported to date. ${ }^{(1)}$ IFFI is usually self-limiting and resolves spontaneously. Most patients improve with conservative management and surgical intervention is not required. ${ }^{(2)}$

The falciform ligament is a double-layered peritoneal fold that attaches the anterior part of the liver to the ventral abdominal wall and inferior diaphragmatic surface. It is a useful anatomical landmark separating the left lobe of the liver into lateral and medial segments. It contains the ligamentum teres, obliterated umbilical vein and extraperitoneal fat (or fatty appendages). The arterial supply to the falciform ligament is via a vessel originating from the left inferior phrenic artery and middle segmental artery of the liver. ${ }^{(4)}$ The veins of the falciform ligament drain into the left inferior phrenic vein. ${ }^{(4)}$

Focal infarction of the falciform ligament fatty appendage was first described on imaging in 2001. ${ }^{(5)}$ It is attributed to torsion of the fat appendage of the falciform ligament and thrombosis of its central vein, leading to fat necrosis and infarction. ${ }^{(5)}$ Patients commonly present with acute abdominal pain that either localises to the epigastrium or right upper quadrant of the abdomen. These symptoms closely mimic other causes of acute abdomen, such as acute cholecystitis. Despite severe abdominal pain, laboratory tests are usually normal or reveal mild inflammatory changes.

An unambiguous diagnosis of focal infarction of the falciform ligament fatty appendage can be made with CT. CT images typically demonstrate a focal area of fat stranding with



Fig. 2 Axial contrast-enhanced CT image of the abdomen and pelvis in a patient shows an ovoid-encapsulated fat inflammation, surrounding inflammatory changes and the 'central dot' sign anterior to the descending colon (arrow), in keeping with epiploic appendagitis.

a thin peripheral rim of high attenuation (the aforementioned 'hyperattenuating rim' sign) in the vicinity of the falciform ligament. This is explained by torsion of the fatty appendage, contributing to fat necrosis manifesting as fat inflammation. The presence of a high-attenuated central focus (the 'central dot' sign) that corresponds to the thrombosed vessel can be helpful to the diagnosis. ${ }^{(2)}$

The diagnosis of focal infarction of the falciform ligament fatty appendage can potentially be missed by inexperienced radiologists if only axial $\mathrm{CT}$ images are interpreted, because the abnormal findings may be subtle, as depicted in this case. However, the imaging features are often distinct in the sagittal plane. Hence, multiplanar reconstruction and interpretation are recommended if subtle abnormalities are seen around the anterior perihepatic space in the axial CT images. ${ }^{(1)}$

Ultrasonography investigation of focal infarction of the falciform ligament fatty appendage usually demonstrates a hyperechoic, non-compressible, slightly heterogeneous mass at the site of tenderness. ${ }^{(6)}$ There is usually absence of vascular flow on colour Doppler interrogation, which is a helpful feature to differentiate it from other infective or inflammatory conditions such as abscess and phlegmon. ${ }^{(2)}$ Bedside ultrasonography is useful as the first-line investigation to exclude alternative diagnoses such as gallstones and acute cholecystitis.

Epiploic appendages are small outpouchings of the visceral peritoneum that contain adipose tissues and small blood vessels, and are attached to the serosal surface of the colon via a vascular stalk. They measure from $0.5 \mathrm{~cm}$ to $5 \mathrm{~cm}$ in length. Depending on the location of the involved appendages, the clinical presentation can mimic appendicitis and cholecystitis (if located around the right hemicolon) or diverticulitis (if located around the left hemicolon). The CT features of epiploic appendagitis are identical to those of focal infarction of the falciform ligament fatty appendage - encapsulated fat inflammation with a central dot representing a thrombosed vessel. The difference is that for epiploic appendagitis, the site of abnormalities is in close proximity to the colon and more often involves the descending colon $^{(7)}$ (Fig. 2). 


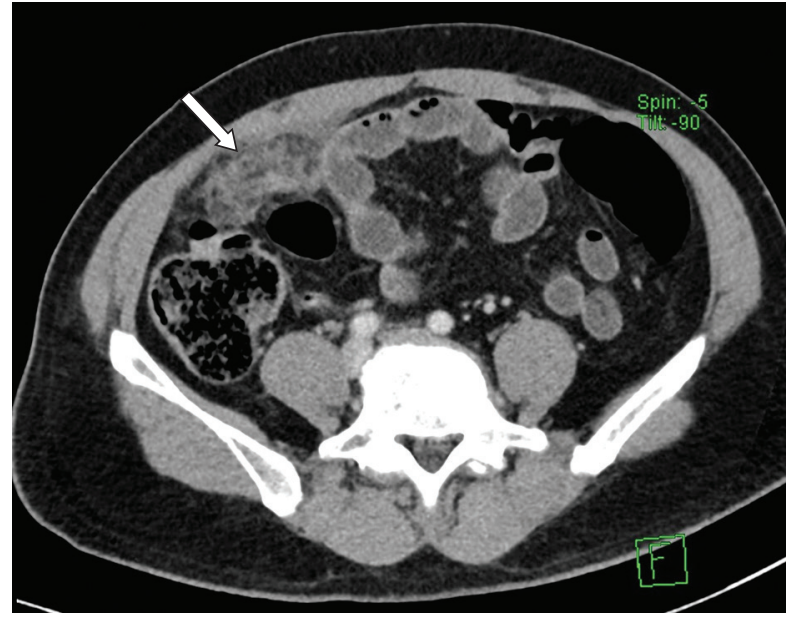

Fig. 3 Axial contrast-enhanced CT image of the abdomen and pelvis in a patient shows focal mass-like fat stranding at the greater omentum in the right lower abdomen (arrow), in keeping with omental infarction.

Omental infarction typically presents on the right side of the abdomen. It is hypothesised that the right lower portion of the omentum has an anomalous and fragile blood supply, which is consequently susceptible to infarction. ${ }^{(8)}$ Clinically, omental infarction can mimic surgical conditions such as acute cholecystitis or appendicitis. On CT, omental infarction is seen as a solitary non-enhancing encapsulated fatty mass (usually $>5 \mathrm{~cm}$ ) with heterogeneous attenuation, often located in the lower right quadrant of the abdomen. It usually lacks the feature of a highattenuating rim, as commonly seen in epiploic appendagitis and focal fat infarction involving the appendages of the falciform ligament (Fig. 3).

In conclusion, focal infarction of the falciform ligament fatty appendage can mimic a variety of conditions that warrant surgical intervention in patients presenting to the ED with acute abdomen. However, radiological diagnosis with CT is often unequivocal. Improved awareness of this condition among radiologists and referring clinicians can serve to avoid unnecessary investigations and surgery.
ABSTRACT A 56-year-old man presented to the emergency department with acute upper abdominal pain and mild leucocytosis. The pain was not relieved by intravenous analgesia. Computed tomography of the abdomen and pelvis demonstrated a focal area of fat stranding with a thin peripheral rim of high attenuation and a punctate central hyperdense dot in the vicinity of the falciform ligament. The diagnosis of focal infarction of the falciform ligament fatty appendage was made based on the imaging findings. The patient responded well to conservative management and was discharged for outpatient follow-up. Focal infarction of the falciform ligament fatty appendage is extremely rare and can mimic various causes of acute abdomen that require emergency surgery. Hence, correct radiological diagnosis is essential to avoid unnecessary surgical intervention. We herein discuss the similarities and differences with other common companion cases such as epiploic appendagitis and omental infarction.

Keywords: acute abdomen, computed tomography, falciform ligament, fatty appendage, intraperitoneal focal fatty infarction

\section{REFERENCES}

1. Indiran V, Dixit R, Maduraimuthu P. Unusual cause of epigastric pain: intraabdominal focal fat infarction involving appendage of falciform ligament - case report and review of literature. GE Port J Gastroenterol 2018; 25:179-83.

2. Coulier B. Contribution of US and CT for diagnosis of intraperitoneal focal fat infarction (IFFI): a pictorial review. JBR-BTR 2010; 93:171-85.

3. Vanderschueren L, Coulier B. Focal fat infarction of the falciform ligament: typical CT appearance of a rare form of intra-peritoneal focal fatty infarction. J Belg Soc Radiol 2019; 103:28.

4. Li XP, Xu DC, Tan HY, Li CL. Anatomical study on the morphology and blood supply of the falciform ligament and its clinical significance. Surg Radiol Anat 2004; 26:106-9.

5. Coulier B, Cloots V, Ramboux A. US and CT diagnosis of a twisted lipomatous appendage of the falciform ligament. Eur Radiol 2001; 11:213-5.

6. Lloyd T. Primary torsion of the falciform ligament: computed tomography and ultrasound findings. Australas Radiol 2006; 50:252-4.

7. Nadida D, Amal A, Ines M, et al. Acute epiploic appendagitis: radiologic and clinical features of 12 patients. Int J Surg Case Rep 2016; 28:219-22.

8. Puylaert JB. Right-sided segmental infarction of the omentum: clinical, US, and CT findings. Radiology 1992; 185:169-72. 


\section{SINGAPORE MEDICAL COUNCIL CATEGORY 3B CME PROGRAMME} (Code SMJ 202001B)

Question 1. Regarding intraperitoneal focal fat infarction (IFFI):

(a) IFFI is a mimicker of acute surgical abdomen.

(b) Focal infarction of the falciform ligament fatty appendage is the most common cause of IFFI.

(c) IFFI is usually self-limiting and resolves spontaneously.

(d) Epiploic appendagitis and omental infarction are uncommon causes of IFFI.

Question 2. Regarding the anatomy of the falciform ligament:

(a) It is a single-layered peritoneal fold that attaches the anterior part of the liver to the ventral abdominal wall and inferior diaphragmatic surface.

(b) It separates the left lobe of the liver into the lateral and medial segments.'

(c) It contains the ligamentum teres, obliterated umbilical vein and fatty appendages.

(d) Its arterial supply is via a vessel originating from the left inferior phrenic artery and middle segmental artery of the liver.

Question 3. Regarding focal infarction of the falciform ligament fatty appendage:

(a) It is attributed to torsion of the fat appendage of the falciform ligament that leads to fat necrosis and infarction.

(b) Patients commonly present with acute abdominal pain at the suprapubic region.

(c) Laboratory tests are usually normal or reveal mild inflammatory changes.

(d) Surgical intervention is the mainstay of management.

Question 4. Regarding imaging of focal infarction of the falciform ligament fatty appendage:

(a) Radiological features on computed tomography (CT) include a focal area of fat stranding with a thin peripheral rim of high attenuation ('hyperattenuating rim' sign) in the vicinity of the falciform ligament.

(b) The CT finding of high-attenuated central focus ('central dot' sign) corresponds to a thrombosed vessel.

(c) Axial CT images are always more sensitive than sagittal images in detecting this pathology.

(d) Ultrasonography usually demonstrates a hyperechoic, slightly heterogeneous mass at the site of tenderness with increased vascularity.

Question 5. Regarding epiploic appendagitis:

(a) Epiploic appendages are small outpouchings of the visceral peritoneum that contain adipose tissues and small blood vessels, and are attached to the serosal surface of the small bowel via a vascular stalk.

(b) The clinical presentation can mimic acute appendicitis and acute cholecystitis.

(c) Radiological features on $\mathrm{CT}$ are identical to those of focal infarction of the falciform ligament fatty appendage, except that it more often affects the descending colon.

(d) It is a self-limiting condition and can be managed conservatively.

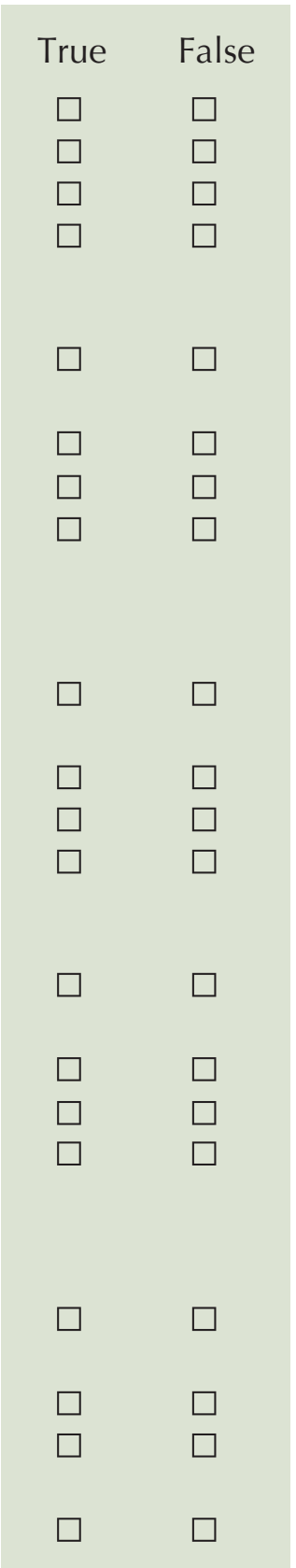

\section{Doctor's particulars:}

Name in full:

MCR no.:

Specialty:

Email:

\section{SUBMISSION INSTRUCTIONS}

Visit the SMJ website: http://www.smj.org.sg/current-issue and select the appropriate quiz. You will be redirected to the SMA login page.

For SMA member: (1) Log in with your username and password (if you do not know your password, please click on 'Forgot your password?'). (2) Select your answers for each quiz and click 'Submit'.

For non-SMA member: (1) Create an SMJ CME account, or log in with your SMJ CME username and password (for returning users). (2) Make payment of SGD 21.40 (inclusive of $7 \%$ GST) via PayPal to access this month's quizzes. (3) Select your answers for each quiz and click 'Submit'.

RESULTS:

(1) Answers will be published online in the SMJ March 2020 issue. (2) The MCR numbers of successful candidates will be posted online at the SMJ website by 12 March 2020. (3) Passing mark is $60 \%$. No mark will be deducted for incorrect answers. (4) The SMJ editorial office will submit the list of successful candidates to the Singapore Medical Council. (5) One CME point is awarded for successful candidates. (6) SMC credits CME points according to the month of publication of the CME article (i.e. points awarded for a quiz published in the January 2020 issue will be credited for the month of January 2020, even if the deadline is in March 2020).

Deadline for submission (January 2020 SMJ 3B CME programme): 12 noon, 5 March 2020. 